\title{
Detection of Noise Level and Resonance Frequency Shifting Through of Principal Component Analysis (PCA) in Simulated Dataset of Band Excitation Scanning Probe Microscopy (BE-SPM)
}

\author{
Carlos Andrés Rosero-Zambrano ${ }^{1}$ and Francy Basante ${ }^{1}$
}

1. South Colombian International Logistics Center, Servicio Nacional de Aprendizaje (National Apprentice Service) - SENA, Tecnoacademia, Tuquerres, Colombia.

Piezoresponse Force Microscopy is a technique to probe the electromechanical coupling at a nanoscale measurement, based on the response detection of the biased tip of the cantilever over the piezoelectric or ferroelectric sample. In the last few years, there have been techniques that were developed based on multifrequency methods such as Dual AC Resonance Tracking (DART) and Band Excitation Piezoresponse Force Microscopy (BE-PFM) [1].

In this work, a numerical model based on a simple harmonic oscillator (SHO) is used to run a simulation and generate an artificial data set that reproduces the behavior observed in BE-PFM to demonstrate the use of the PCA to identify the level of noise that affects the signal in BE-PFM response. This simulated model includes the following independent parameters: voltage modulated with a periodic tip bias $V_{A C}$, inverse piezoelectric constant $d_{33}$, drive frequency $\omega$, contact resonance frequency $\omega_{0}$ and the dimensionless quality factor $Q$, with these parameters the amplitude $A$ is calculated by SHO model [2].

The analysis of a multi-dimensional, spectroscopic-imaging data based on PCA allows to identify through the first three eigenvectors $\left(v_{1}, v_{2}, v_{3}\right)$ characteristics like: average spectrum $\left(v_{1}\right)$, frequency shift $\left(v_{2}\right)$ and peak broadening $\left(v_{3}\right)$. These eigenvectors were extracted by computing the Singular Value Decomposition (SVD), which is a matrix factorization method, used to decompose a matrix into the product of three matrices such that $A=U S V^{T}$ [3], where $A$ is a matrix of all experimental data points to conform the frequency spectra along the surface scanned sample, $S$ and $V$ are matrices with the eigenvalues and eigenvectors respectively used to build the bivariate maps from the first three eigenvectors of $A$ the results are illustrated graphically in Figure 1.

A noise level $\left(Y_{\text {noise }}\right)$ is added ( 0 and 5) with small and high variations of contact resonance frequency (CRF) within the spectroscopic data to examine the effects in the eigenvectors and to explore four different scenarios, to demonstrate the applicability of PCA and SVD for a rapid detection of noise and quantify the contact resonance frequency variations, it shows how to be a powerful tool in understanding the coupling between multivariate analysis methods and material science at nanoscale.

References:

[1] B. J. Rodriguez et al, in "Scanning Probe Microscopy of Functional Materials", (Springer, New York) p. 491.

[2] S. Jesse and S. V Kalinin, Nanotechnology 20(8) (2009), p. 85714.

[3] M. H. Trauth in "MATLAB ${ }^{\circledR}$ Recipes for Earth Sciences, Berlin, Heidelberg”, (Springer, Berlin) p. 375. 


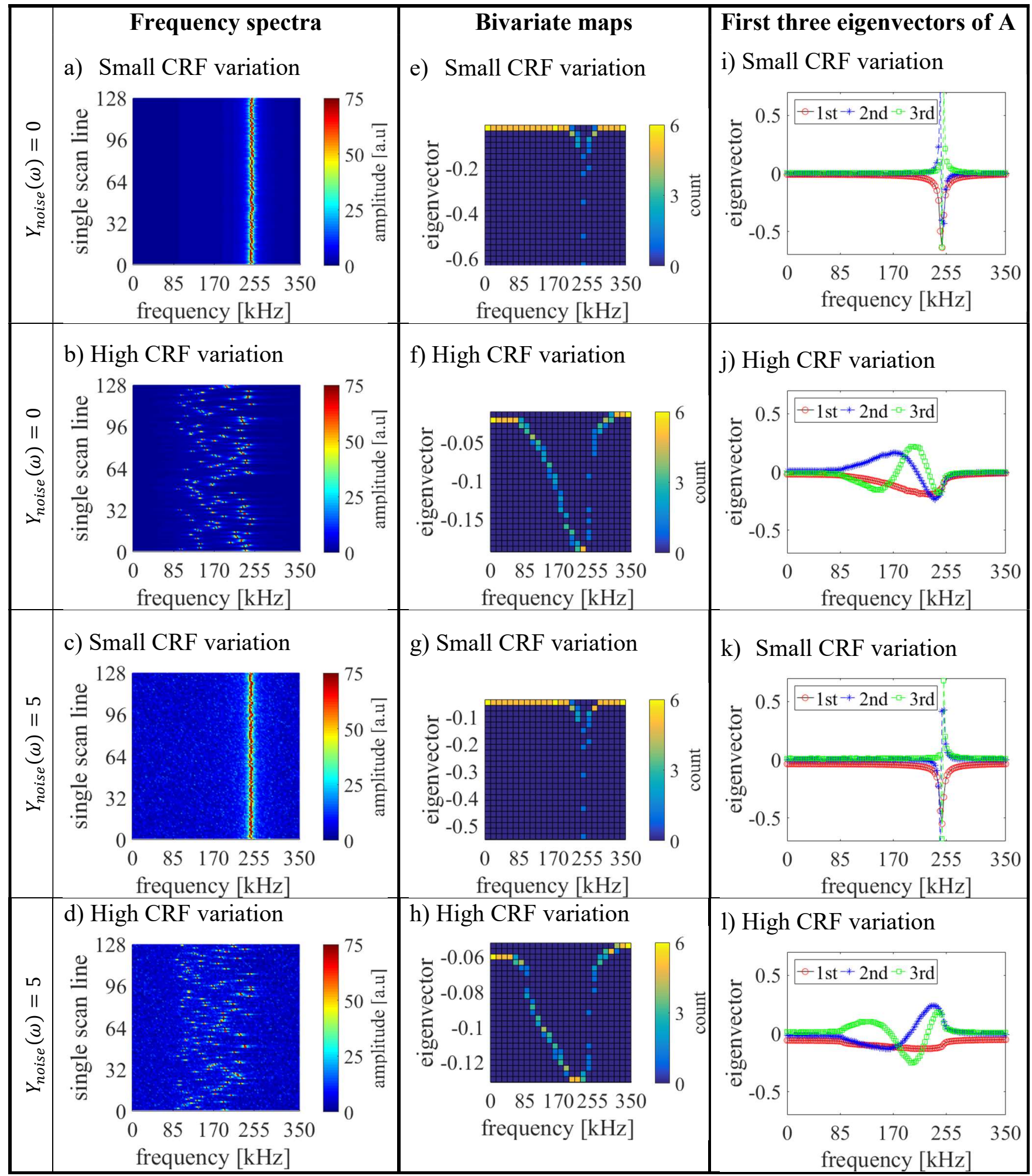

Figure 1. (a), (c) Frequency spectra with small variations in contact resonance at single line scan. (b), (d) Frequency spectra with high variations in contact resonance at single line scan. (a), (b) Simulation without noise added $Y_{\text {noise }}(\omega)=0$. (c), (d) Simulation with noise level added $Y_{\text {noise }}(\omega)=5$. (e) $-(\mathrm{h})$ Bivariate maps to four scenarios small and high variations in contact resonance. (i) - (1) Overall shape of the first three eigenvectors of all experimental data points in matrix A. 